\title{
Microwave and laser spectroscopy of carbon chains and rings
}

\author{
Michael C. McCarthy ${ }^{a, b}$ and Patrick Thaddeus ${ }^{a, b}$ \\ a Harvard-Smithsonian Center for Astrophysics, 60 Garden Street, Cambridge, MA 02138, USA \\ ${ }^{b}$ Division of Engineering and Applied Sciences, 29 Oxford Street, Cambridge, MA 02138, USA
}

Received 16th August 2000

First published as an Advance Article on the web 5th April 2001

The interstellar gas and the gas ejected into space by cool stars are now known to contain a rich collection of molecules. Most of these are organic compounds, some of which are familiar to the terrestrial chemist and can be found in a standard chemical stockroom, but many are entirely new molecules which have been detected and identified in space for the first time. Most of the nearly 100 polyatomic molecules so far detected in astronomical sources by radio telescopes are highly unsaturated carbon chains, a structure which is explosively unstable at even moderate density, and therefore difficult to study spectroscopically on Earth. For this reason laboratory detection has until quite recently lagged well behind the astronomical discovery of many carbon chains. The application of Fourier transform microwave and laser cavity ringdown absorption spectroscopy to supersonic molecular beams has largely overcome this difficulty, yielding in this laboratory during the past four years more than 75 new reactive molecules, including long carbon chains, chains attached to rings, silicon-carbon rings, and several protonated molecular ions. All or nearly all of these are candidates for astronomical discovery because they are highly polar and are close in structure and composition to molecules which have already been discovered in space. Seven have already been found with large radio telescopes, six as a result of the laboratory work described here. These discoveries include the largest interstellar molecule $\mathrm{HC}_{11} \mathrm{~N}$, the largest ring, $\mathrm{SiC}_{3}$, and the largest positive ion, $\mathrm{HC}_{3} \mathrm{NH}^{+}$. One of our most interesting recent laboratory discoveries is the laser detection of a strong molecular band at $\lambda \mathbf{4 4 2 9}$, the wavelength of the strongest and best known diffuse interstellar band.

\section{Introduction}

In space as on Earth, the ambiguity of carbon in bonding and its unique ability to form both single and multiple bonds give rise to a chemistry of great subtlety and complexity. In the interstellar gas and in the gas being ejected into space from cool stars, 123 molecules have now been detected and identified (Fig. 1), mainly by radio telescopes with high spectral resolution receivers able to resolve the sharp rotational emission lines characteristic of molecules in cold, low density gas excited by collisional impact. The simple astronomical molecules are both inorganic and organic in composition, but all those with more than five atoms are organic, and the majority of these are carbon chains with linear heavy atom backbones of highly unsaturated carbon (generally unsymmetrical in structure, as Fig. 1 shows, to provide the permanent electric dipole moment
Michael C. McCarthy received a BSC in Chemistry at the University of Alaska, and a PhD in physical chemistry from MIT in 1992 with R. W. Field. He then moved to the HarvardSmithsonian Center for Astrophysics as a Center Fellow with Patrick Thaddeus. He remained here as a member of the scientist staff and in 1997 was appointed to his present position as the Yoram Avni Distinguished Research Astronomer. His research interests include the spectroscopy of known and postulated astronomical carbon chains, carbon rings, and

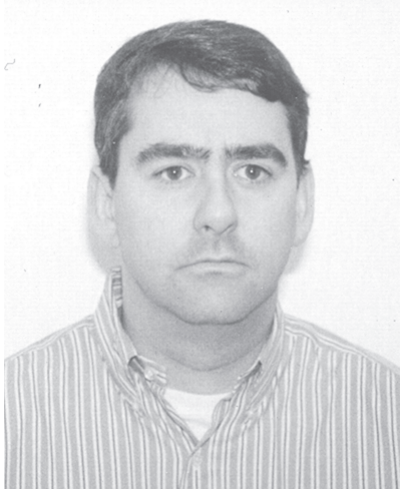
carbon clusters; the chemistry and physics of the interstellar medium; and molecular radio astronomy.

Michael C. McCarthy

Patrick Thaddeus was born in 1932 and received his education at the University of Delaware (BS 1953), the University of Oxford (MA 1955), and Columbia University (PhD in physics 1960, under the supervision of C. H. Townes). He worked until 1986 in New York on the staff of the Goddard Institute for Space Studies and as an adjunct Professor of Physics of Columbia. In 1986 he joined the faculties of the Harvard-Smithsonian Center for Astrophysics and the Division of Engineering and Applied Science at Harvard; he is currently the Robert Wheeler Willson

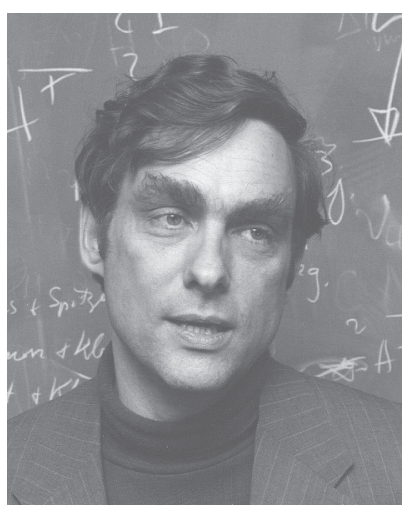

Patrick Thaddeus Professor in the Astronomy Department. His research interests include the radio astronomy of interstellar and circumstellar molecules, the structure of our own Galaxy and its nearest neighbors, and the laboratory investigation of molecules which are candidates for astronomical detection. He is the author or co-author of nearly 300 research papers and reviews. 
required for radio detection). It is one of the paradoxes of interstellar chemistry that unsaturated carbon should be so conspicuous in a regime where hydrogen is the dominant chemically active element by more than three orders of magnitude.

It is thought that many of the molecules in Fig. 1 are produced by gas-phase ion-molecule reactions which occur rapidly even at the low temperature (less than $100 \mathrm{~K}$ ) characteristic of the interstellar gas. Ion-molecule processes, for example, predict the existence of many reactive molecules and isomers whose abundances are far from those that would be predicted from equilibrium considerations alone, including polyatomic ions such as $\mathrm{HCO}^{+}$, and energetic isomers such as $\mathrm{HNC}$ and the cumulene carbene $\mathrm{H}_{2} \mathrm{C}_{4}$. The abundance of carbon chains, in particular, is attributable to the formation of $\mathrm{C}^{+}$by the reaction $\mathrm{CO}+\mathrm{He}^{+} \rightarrow \mathrm{C}^{+}+\mathrm{O}+\mathrm{He},{ }^{1}$ and by the ionization of atomic carbon by the hard UV photons from hot stars. Recent laboratory studies indicate that atomic carbon efficiently reacts with a variety of stable hydrocarbons at room temperature and below, implying that neutral-neutral reactions may also play a role in the formation of longer carbon chains. ${ }^{2,3}$

Most of the polyatomic molecules in Fig. 1 have been discovered in molecular clouds, the dense component of the interstellar gas where the hydrogen is mainly $\mathrm{H}_{2}$, but a few have been found in the lower density so-called "diffuse" clouds which are mainly atomic $\mathrm{H}$, and many others in circumstellar shells around evolved stars. Molecular clouds have densities from $10^{2}$ to $10^{6} \mathrm{~cm}^{-3}$ and temperatures that are generally under $50 \mathrm{~K}$ (although higher in the vicinity of newly formed hot stars); diffuse clouds have densities from 10 to $100 \mathrm{~cm}^{-3}$ and are generally hotter than molecular clouds, with temperatures from 50 to $150 \mathrm{~K}$; the circumstellar ejecta where molecules can form and survive have densities of roughly $10^{5} \mathrm{~cm}^{-3}$ and temperatures of $20 \mathrm{~K}$. In all three types of objects dust grains formed from the cosmic nonvolatile elements are present in a fairly constant proportion by mass. Molecular clouds, as a result, tend to be highly opaque, diffuse clouds fairly transparent, and circumstellar shells of intermediate opacity. In all three locations, many rotational levels of polyatomic molecules are generally populated, but the populations of excited vibrational and electronic levels are generally negligible.

An enigmatic family of optical diffuse interstellar bands (DIBs) have long been observed in the diffuse clouds. ${ }^{4}$ [There is an unfortunate ambiguity here: the bands have traditionally been termed 'diffuse' not because of the clouds in which they are observed, but rather because their profiles are wide and generally featureless, nearly all lacking appreciable rotational structure.] More than 125 are now known in the visual and near infrared, ${ }^{5}$ but despite much work over the past 50 years, the carrier of none has been positively identified. [A possible exception is the carbon chain anion $\mathrm{C}_{7}{ }^{-}$, which may be responsible for four or five diffuse bands in the region 560-630 $\mathrm{nm},{ }^{6}$ but recent astronomical observations by McCall et al. ${ }^{7}$ cast doubt on this identification.] The DIBs are almost exclusively seen in absorption against nearby fairly bright stars, but several may occur in emission toward one peculiar source, the Red Rectangle. ${ }^{8}$ Although in the past it has been argued that the DIBs originate in the interstellar dust grains, there is now much evidence that they are produced by molecules in the gas phase, some plausible carriers being carbon chains, ${ }^{9}$ polycyclic aromatic hydrocarbons, ${ }^{10}$ and fullerenes. ${ }^{11}$ The conspicuous presence of linear carbon elsewhere in the interstellar gas in the dense molecular component is an obvious merit of the carbon chain hypothesis.

The first molecules detected in space with radio telescopes, $\mathrm{OH}, \mathrm{H}_{2} \mathrm{O}, \mathrm{NH}_{3}$, and then $\mathrm{H}_{2} \mathrm{CO}$ were well known to spectroscopists prior to their astronomical discovery. Many of the molecules subsequently detected in space, however, were highly reactive species, generally unknown or unfamiliar to the laboratory chemist or physicist. Because many of these are

Known Interstellar and Circumstellar Molecules (July 2000)

\begin{tabular}{|c|c|c|c|c|c|c|c|}
\hline 2 & 3 & 4 & 5 & $\begin{array}{c}\text { mber of Atoms } \\
6\end{array}$ & 7 & 8 & 9 \\
\hline $\mathbf{H}_{2}$ & $\mathrm{H}_{2} \mathrm{O}$ & $\mathbf{N H}_{3}$ & $\mathrm{SiH}_{4}$ & $\mathrm{CH}_{3} \mathrm{OH}$ & $\mathrm{CH}_{3} \mathrm{CHO}$ & $\mathrm{CH}_{3} \mathrm{CO}_{2} \mathrm{H}$ & $\mathrm{CH}_{3} \mathrm{CH}_{2} \mathrm{OH}$ \\
\hline OH & $\mathrm{H}_{2} \mathbf{S}$ & $\mathrm{H}_{3} \mathrm{O}^{+}$ & $\mathrm{CH}_{4}$ & $\mathrm{NH}_{2} \mathrm{CHO}$ & $\mathrm{CH}_{3} \mathrm{NH}_{2}$ & $\mathrm{HCO}_{2} \mathrm{CH}_{3}$ & $\left(\mathrm{CH}_{3}\right)_{2} \mathrm{O}$ \\
\hline so & $\mathrm{SO}_{2}$ & $\mathrm{H}_{2} \mathrm{CO}$ & СНООН & $\mathrm{CH}_{3} \mathrm{CN}$ & $\mathrm{CH}_{3} \mathrm{CCH}$ & $\mathrm{CH}_{3} \mathrm{C}_{2} \mathrm{CN}$ & $\mathrm{CH}_{3} \mathrm{CH}_{2} \mathrm{CN}$ \\
\hline $\mathrm{SO}^{+}$ & $\mathrm{HN}_{2}^{+}$ & $\mathrm{H}_{2} \mathrm{CS}$ & $\mathrm{HC} \equiv \mathrm{CCN}$ & $\mathrm{CH}_{3} \mathrm{NC}$ & $\mathrm{CH}_{2} \mathrm{CHCN}$ & $\mathrm{C}_{7} \mathrm{H}$ & $\mathrm{H}(\mathrm{C} \equiv \mathrm{C})_{3} \mathrm{CN}$ \\
\hline SiO & HNO & HNCO & $\mathrm{CH}_{2} \mathrm{NH}$ & $\mathrm{CH}_{3} \mathrm{SH}$ & $\mathrm{HC}_{4} \mathrm{CN}$ & $\mathbf{H}_{2} \mathbf{C}_{6}$ & $\mathrm{H}(\mathrm{C} \equiv \mathrm{C})_{2} \mathrm{CH}_{3}$ \\
\hline Sis & $\mathrm{SiH}_{2} ?$ & HNCS & $\mathrm{NH}_{2} \mathrm{CN}$ & $\mathrm{C}_{5} \mathrm{H}$ & $\mathrm{C}_{6} \mathrm{H}$ & & $\mathrm{C}_{8} \mathrm{H}$ \\
\hline NO & $\mathrm{NH}_{2}$ & CCCN & $\mathrm{H}_{2} \mathrm{CCO}$ & $\mathrm{HC}_{2} \mathrm{CHO}$ & c- $\mathrm{CH}_{2} \mathrm{OCH}_{2}$ & & \\
\hline NS & $\mathbf{H}_{3}^{+}$ & $\mathrm{HCO}_{2}^{+}$ & $\mathrm{C}_{4} \mathrm{H}$ & $\mathbf{C H}_{2}=\mathrm{CH}_{2}$ & $\mathbf{C}_{7}^{-} ?$ & & 10 \\
\hline $\mathrm{HCl}$ & NNO & $\mathrm{CCCH}$ & c- $\mathrm{C}_{3} \mathrm{H}_{2}$ & $\mathrm{H}_{2} \mathrm{CCCC}$ & & & \\
\hline $\mathrm{NaCl}$ & HCO & c-CCCH & $\mathrm{CH}_{2} \mathrm{CN}$ & $\mathrm{HC}_{3} \mathrm{NH}^{+}$ & & & $\mathrm{CH}_{3} \mathrm{COCH}_{3}$ \\
\hline $\mathrm{KCl}$ & $\mathrm{HCO}^{+}$ & ccco & $\mathbf{C}_{5}$ & $\mathbf{C}_{5} \mathbf{N}$ & / & & $\mathrm{CH}_{3}(\mathrm{C} \equiv \mathrm{C})_{2} \mathrm{CN} ?$ \\
\hline $\mathrm{AlCl}$ & ocs & cCCs & $\mathrm{SiC}_{4}$ & $\mathrm{C}_{5} \mathbf{S} ?$ & & 120 & \\
\hline AIF & $\mathrm{CCH}$ & $\mathrm{HCCH}$ & $\mathrm{H}_{2} \mathrm{CCC}$ & & & & 11 \\
\hline PN & $\mathrm{HCS}^{+}$ & $\mathrm{HCNH}^{+}$ & HCCNC & & & 100 웅 & \\
\hline $\operatorname{SiN}$ & c-SiCC & $\mathrm{HCCN}$ & HNCCC & & & อี & $\mathrm{H}(\mathrm{C} \equiv \mathrm{C})_{4} \mathrm{CN}$ \\
\hline NH & $\mathrm{cco}$ & $\mathrm{H}_{2} \mathrm{CN}$ & $\mathrm{H}_{3} \mathrm{CO}^{+}$ & & & 80 อ & \\
\hline $\mathrm{CH}$ & CCs & c-SiC 3 & & & & ○ & 13 \\
\hline $\mathrm{CH}^{+}$ & $\mathbf{C}_{3}$ & $\mathrm{CH}_{3}$ & & & & $60 \stackrel{\text { 点 }}{\gtrless}$ & \\
\hline $\mathrm{CN}$ & MgNC & $\mathrm{CH}_{2} \mathrm{D}^{+} ?$ & & & & อ & $\mathrm{H}(\mathrm{C} \equiv \mathrm{C})_{5} \mathrm{CN}$ \\
\hline $\mathrm{CO}$ & $\mathrm{NaCN}$ & & & & & $40 \stackrel{\frac{2}{8}}{2}$ & \\
\hline CS & $\mathrm{CH}_{2}$ & & & & & 40 & \\
\hline $\mathbf{C}_{2}$ & $\mathrm{MgCN}$ & & & & & \& & \\
\hline Sic & HOC $^{+}$ & $\begin{array}{l}\mathrm{CH}^{+} \\
\mathrm{CH}^{+},\end{array}$ & & & & 20 & Total: 123 \\
\hline CP & $\mathrm{HCN}$ & $\mathrm{CN}$ & $\mathrm{OH}$ & & & & \\
\hline $\mathrm{CO}^{+}$ & HNC & $\longdiv { 1 }$ & 1 & 1 & 1 & & \\
\hline HF & $\begin{array}{l}\text { SiCN } \\
\text { KCN? }\end{array}$ & 1940 & 60 & $\begin{array}{l}70 \\
\text { Year }\end{array}$ & 2000 & & \\
\hline
\end{tabular}

Fig. 1 Molecules identified in the interstellar gas and in circumstellar shells, ranked by number of atoms. Insert-Cumulative total by year since 1940 . 
carbon chains which are unstable at terrestrial densities, tending to explosively polymerize to disordered carbon with a more or less graphitic structure, laboratory detection has proved challenging, and, as a result, has often lagged rather than preceded astronomical discovery. Recent advances here and in other laboratories, however, suggest that the means are now at hand to overcome this difficulty, and that any polar molecule detected in space can now be found in the laboratory with present techniques or with a moderate refinement of them. Two have been found to be particularly powerful when applied to a supersonic molecular beam: Fourier transform microwave (FTM) spectroscopy and cavity ringdown absorption spectroscopy (CRDS). Together in our laboratory they have resulted in the detection of more than 75 reactive carbon molecules (Fig. 2), including chains, ring-chains, and rings; 13 are closed-shell polyynes, 33 are radicals, 27 are carbenes, and two are ions. Of these, 71 are polar molecules whose rotational spectra have been detected by FTM spectroscopy at centimeter wavelengths, and four are non-polar molecules whose optical spectra have been detected by CRDS at visible wavelengths. As discussed below, a fifth molecule detected by CRDS, of still uncertain composition and structure, gives rise to a strong molecular band at $4429 \AA$, precisely the wavelength of the strongest and best known DIB.

For most of our new discoveries, the laboratory spectroscopy is now ahead of the observational astronomy, and astronomical searches, typically inefficient and costly in telescope time when frequencies are uncertain, can now be based on very precise spectral line frequencies. The large number of laboratory detections also provides much new data for the next generation of radio and optical astronomical investigations. Because of limited space it is not possible to give much here in the way of experimental details, or to present more than a brief survey of the flood of new data. Instead, we briefly describe the laboratory detection of several of the new molecules, and discuss joint astronomical and laboratory investigations that have led to recent astronomical discoveries.

\section{Approach and experimental techniques}

\subsection{FTM spectroscopy}

The application of FTM spectroscopy to supersonic molecular beams ${ }^{12,13}$ is particularly effective in the study of the large reactive carbon chains found in space, because the rotational spectra of these molecules are greatly simplified at the low rotational temperature of a few degrees Kelvin that is readily achieved in a supersonic expansion. In our spectrometer, as Fig. 3 shows, reactive molecules are created by a small low-current dc discharge in the throat of the expansion nozzle as a gas pulse consisting of a small amount of organic vapor heavily diluted (99\% or more) in an inert gas (Ar or $\mathrm{Ne}$ ) expands from a commercial pulsed valve. Free expansion from the nozzle forms in a short distance an approximately Mach 2 supersonic beam with a rotational temperature approaching $1 \mathrm{~K}$. As the beam passes through a high- $Q$ Fabry-Perot microwave cavity, a rotational transition resonant with one of the modes of the cavity is excited coherently by a short pulse of resonant microwave radiation; the microwaves almost immediately die away, and line radiation by the coherently rotating molecules is then detected by a sensitive microwave receiver. The Fourier transform of the free induction decay yields the power spectrum. For the axial beam illustrated in Fig. 3-the configuration adopted for nearly all our experiments because it gives the best signal-to-noise and resolution-the line shape is double peaked, owing to the two Doppler components which result from decomposition of the standing mode of the FabryPerot cavity into two traveling waves, one parallel to the supersonic beam, the other antiparallel. In practice, this peculiar lineshape is usually only a minor inconvenience, and the effective spectral resolution of the spectrometer is determined by the small width of the individual Doppler peaks.

The success of our centimeter-wave device is principally the result of five factors: (1) the high intrinsic sensitivity of the technique, particularly when the molecular beam is directed along the axis of the Fabry-Perot cavity; 14 (2) careful optimization of the geometry and electrical characteristics of the discharge and nozzle; (3) the general use of unstable and explosive precursor compounds; (4) refinements to the microwave receiver, including careful electromagnetic coupling of the receiver to the Fabry-Perot cavity and cooling of the cavity mirrors and the first-stage of the receiver amplifier to $77 \mathrm{~K}$ with liquid nitrogen; and (5) computer control which allows automatic scanning over wide frequency ranges and rapid data transfer and analysis. Together these factors have allowed us to attain an improvement in sensitivity of one to two orders of magnitude over that of the free-space millimeter-wave absorption spectrometer with which all our laboratory discoveries were made prior to 1996 .

\subsection{CRD spectroscopy}

Many of the new laboratory molecules shown in Fig. 2 are plausible carriers of the diffuse interstellar bands, and it is for the optical study of the electronic spectra of those detected in the radio band and similar molecules that a laser spectrometer was recently constructed in this laboratory. The radio spectroscopy is complete in the sense that the identity of each molecule is quite secure and precise constants for the rotational spectra of each have been determined, but the electronic spectra of nearly all is unknown. For a given molecular density, laser spectroscopy is generally much more sensitive than rotational spectroscopy, so laser detection should be possible for many. (The red and violet bands of $\mathrm{CN}$, for example, were first identified in $1932,{ }^{15}$ but it was 45 years later that the rotational spectrum of this readily produced radical was observed by millimeter-wave absorption spectroscopy). ${ }^{16}$ Most of the molecules shown in Fig. 2 have conjugated structures, similar in their properties to organic dyes, with similarly strong electronic transitions that make them good candidates for the carriers of the DIBs. The specific abundances in our molecular beam of these molecules are known fairly accurately from the FTM measurements, and most are adequate for laser detection $\left(\sim 10^{9} \mathrm{~cm}^{-3}\right)$.

CRDS is expected to be the technique of choice because most of the larger carbon molecules shown in Fig. 2 probably fail to fluoresce, or do so only weakly, so detection by the very sensitive technique of laser-induced fluorescence is prohibited. The same mechanism of rapid internal conversion which quenches fluorescence in these molecules may also provide for the wide, generally featureless absorption profiles of most diffuse bands. ${ }^{9}$ A schematic of our laser cavity ringdown spectrometer is shown in Fig. 3. The discharge source for reactive molecules is nearly identical to the one employed in the FTM experiment.

CRDS is long path, sensitive absorption spectroscopy ${ }^{17,18}$ achieved by measuring the rate of absorption of a light pulse confined within a highly reflective optical cavity. It has become a practical spectroscopic tool because of its ease and convenience, and because of the commercial availability of fairly inexpensive mirrors with reflectivities of order $99.99 \%$, accomplished by the deposition of several tens of layers of dielectric 
material; mirrors in the visual, several centimeters in diameter, typically cost \$1000-2000 a pair, and are highly reflective over a bandwidth of $15 \%$. CRDS is more sensitive than conventional absorption spectroscopy with a pulsed dye laser by about four orders of magnitude, meaning that relative absorptions as small as $\Delta I / I \sim 10^{-7}$ can be detected.

\section{Polyynes}

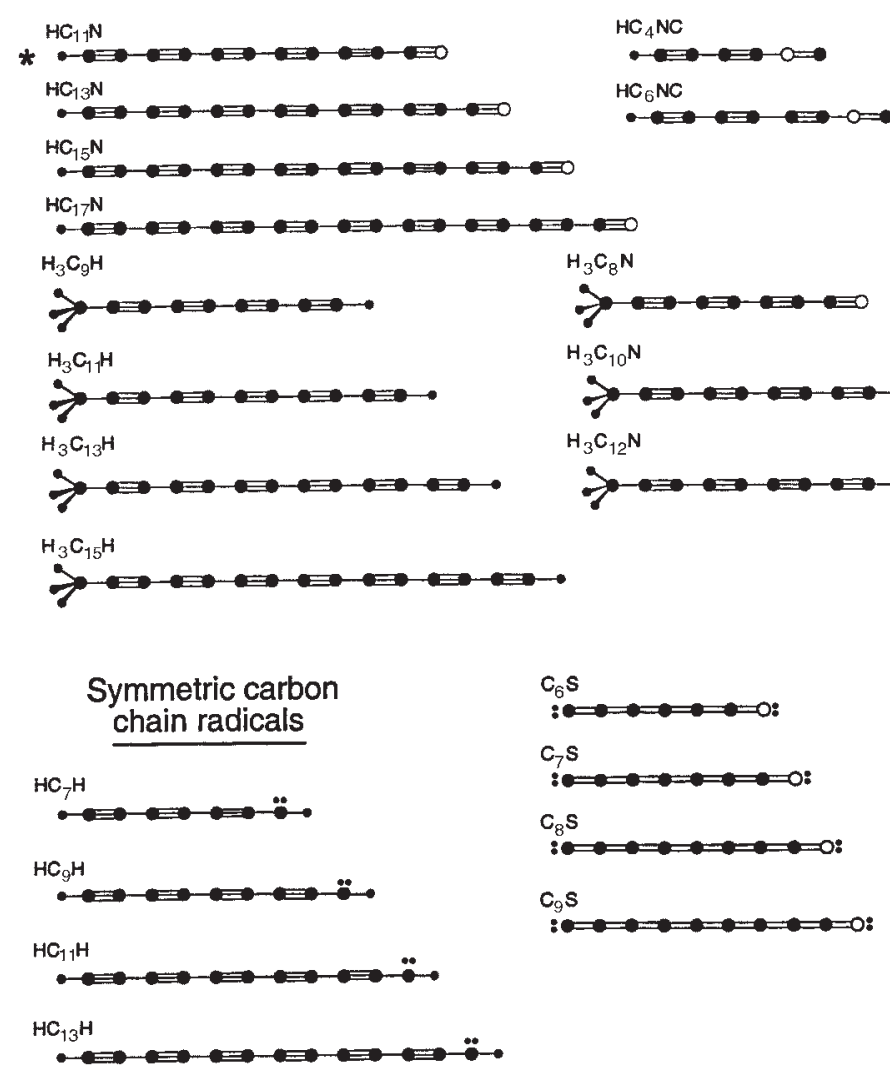

Silicon carbon chains and rings
*
(S)

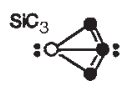

$\mathrm{Sic}_{3}$

$: 0=0$

$\mathrm{SiC}_{2} \mathrm{H}$

$: 0=0$.

$\mathrm{SiC}_{4} \mathrm{H}$

$: 0=0$
$\mathrm{Sic}_{5}$

$0=0=0:$

$\mathrm{SiC}_{6}$

$: 0=\bullet \because=\bullet$

$\mathrm{SiC}_{7}$

$: \because \because \because \because \because$

$\mathrm{SiC}_{8}$

$0=0=0$

$\mathrm{SiC}_{5} \mathrm{H}$

$: 0=0=-$

$\mathrm{SiC}_{6} \mathrm{H}$

$: 0=0 \Leftrightarrow=0$

SiCN

$*: 0=0$

$\mathrm{H}_{2} \mathrm{CCSi}$

\section{Sulfur carbon chains}
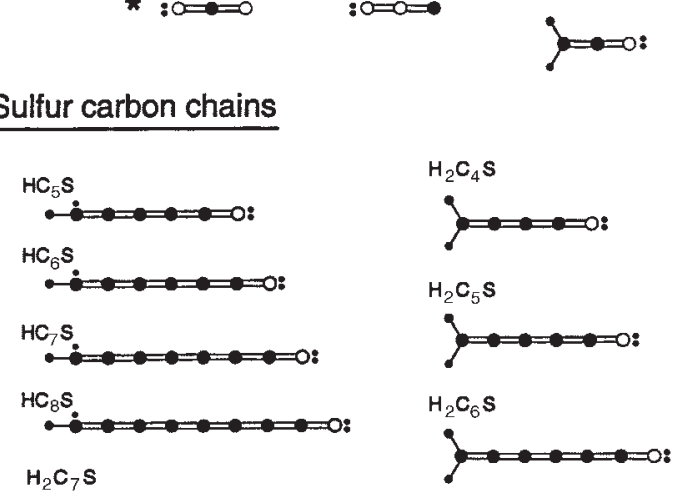

$\xi=0=0=0$

\section{Carbon Chain Radicals}

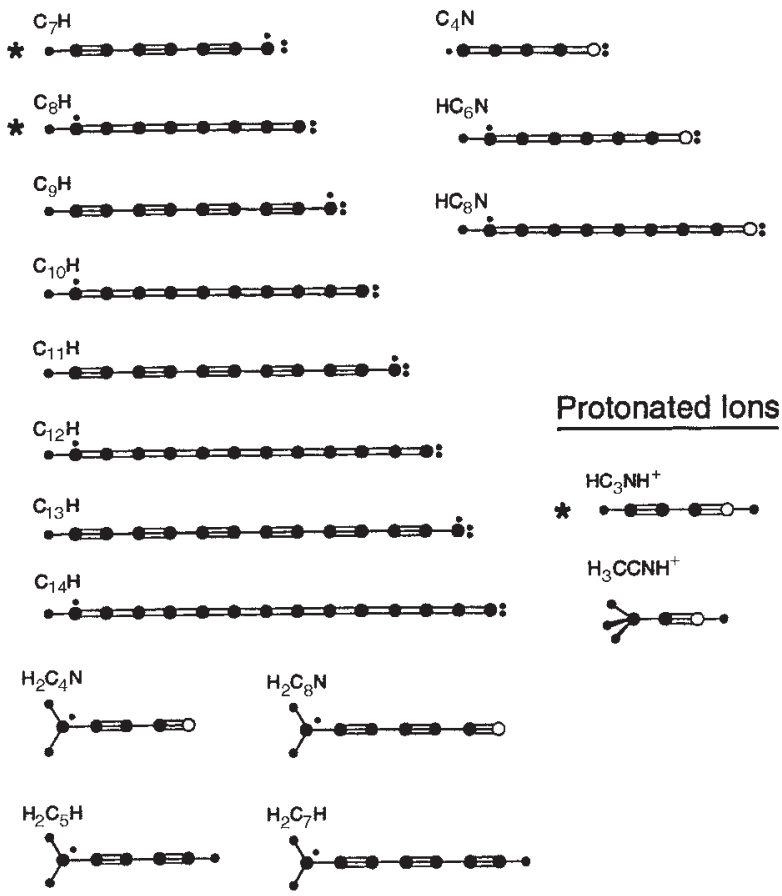

\section{Carbenes}
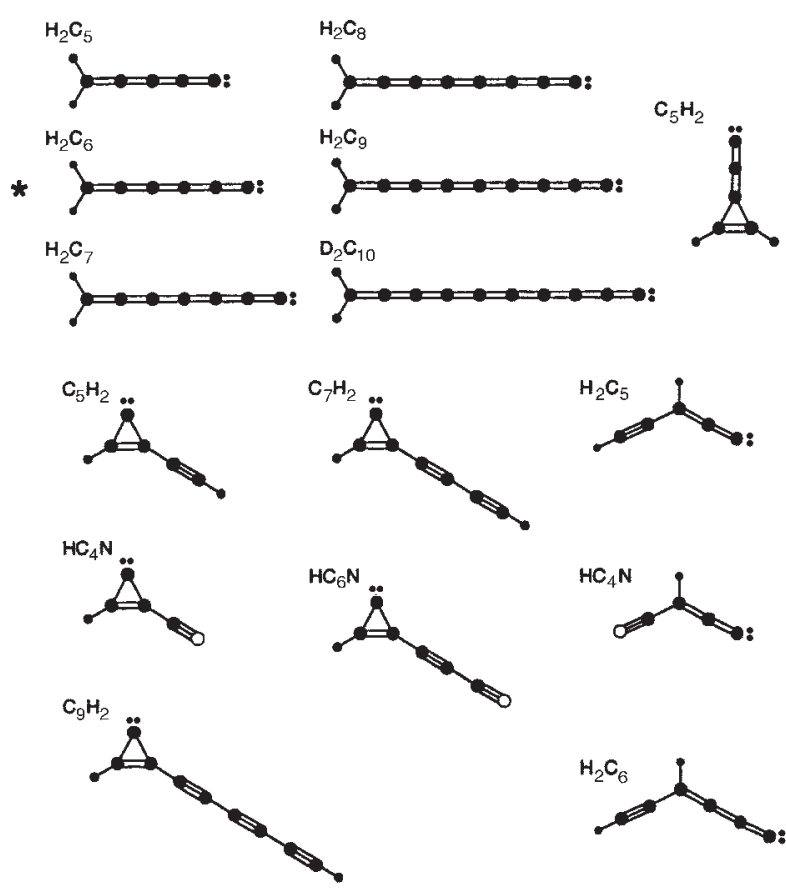

Fig. 2 The seventy-five molecules discovered here during the past four years. Open circles denote hetero atoms, either N, Si, or S. Asterisks $\left(^{*}\right)$ indicate those molecules already detected in space, all but one $\left(\mathrm{HC}_{3} \mathrm{NH}^{+}\right)$on the basis of the present laboratory work. The laboratory astrophysics of all is complete in the sense that the radio spectra (including hyperfine structure where it exists) have been measured directly or can be calculated to better than $1 \mathrm{ppm}$. 
FTM Spectrometer

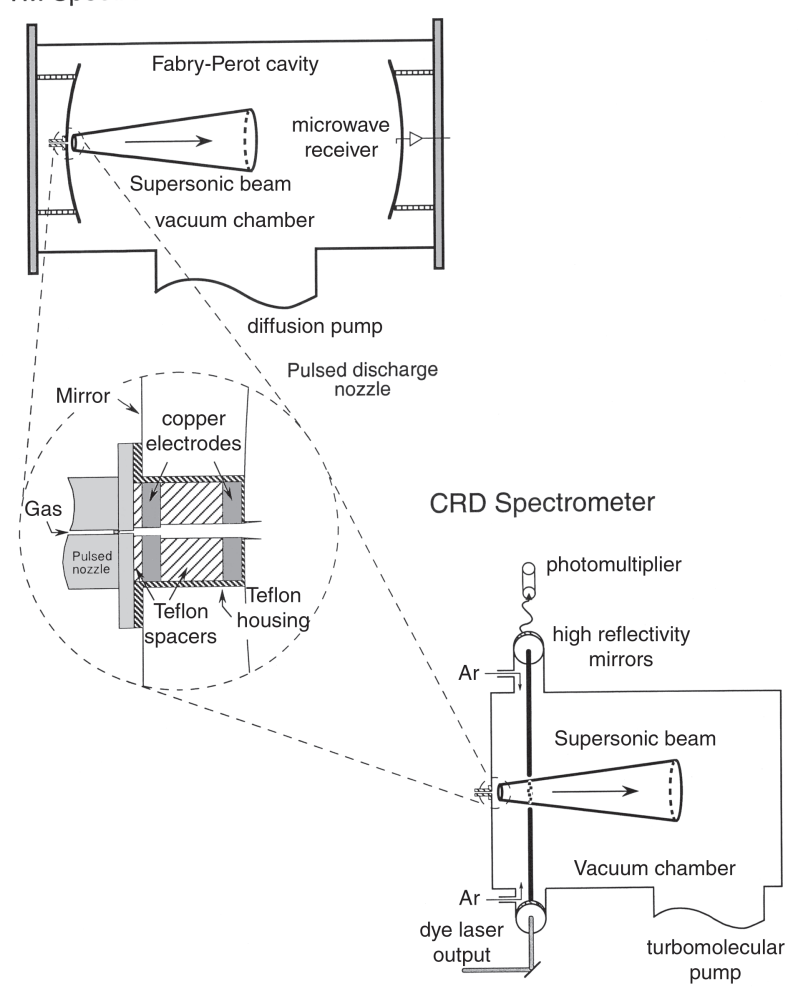

Fig. 3 Schematic diagram of the FTM and CRD spectrometers. In both experiments reactive molecules are produced in a small low-current dc discharge in the throat of a nozzle through a stream of a precursor vapor (diacetylene, cyanoacetylene, acetylene, silane, etc., diluted $99 \%$ or more with $\mathrm{Ne}$ or Ar), just prior to supersonic expansion to about Mach 2 in a large vacuum chamber. The stagnation pressure of the gas behind the valve is 1-3 atm, the discharge potential is $1000-1800 \mathrm{~V}$, and the length of the gas pulse is typically $200-350 \mu \mathrm{s}$. The diameter of the opening orifice of the nozzle immediately following the valve is $1 \mathrm{~mm}$. A pulsed valve working $6-10 \mathrm{~Hz}$, open less than $1 \%$ of the time, is required to keep the pressure in the chamber low enough to form a good molecular beam.

\section{Recent results}

\subsection{The long cyanopolyyne chain $\mathrm{HC}_{17} \mathrm{~N}$}

The longest carbon chain so far detected in our laboratory is a large molecule by the standards of known astronomical chains: $\mathrm{HC}_{17} \mathrm{~N}$ with 19 atoms and a molecular weight of $219 \mathrm{amu},{ }^{19}$ nearly three times the molecular weight of glycine, the simplest amino acid. $\mathrm{HC}_{17} \mathrm{~N}$ and other long chain cyanopolyynes are produced best in our discharge with a mixture of cyanoacetylene $\left(\mathrm{HC}_{3} \mathrm{~N}\right)$ and diacetylene $\left(\mathrm{HC}_{4} \mathrm{H}\right)$ in Ne. Like other closedshell linear molecules, the radio spectrum of $\mathrm{HC}_{17} \mathrm{~N}$ can be calculated to high precision from two free parameters: $v_{J \rightarrow J-1}$ $=2 B J-4 D J^{3}$, where $J$ is the angular momentum quantum number for the upper level of the transition, and $B$ and $D$ are the rotational constant and the leading centrifugal distortion constant. Shown in Fig. 4 is an energy level diagram of the lower rotational levels of $\mathrm{HC}_{17} \mathrm{~N}$ and the transitions that have been measured, along with a sample line. The lower-lying rotational transitions below $1 \mathrm{GHz}$ are expected to have resolvable nitrogen quadrupole hfs, but for the high- $J$ transitions we have observed this hyperfine structure (hfs) has collapsed and is too small to observe.

As found for other closed-shell chains, the rotational constants of the longer cyanopolyynes are remarkably well predicted by extrapolation. ${ }^{20}$ The predicted constants agree with those measured to within a few parts in 100,000 for both $\mathrm{HC}_{15} \mathrm{~N}$ and $\mathrm{HC}_{17} \mathrm{~N}$, so that at $5 \mathrm{GHz}$ (where $\mathrm{HC}_{17} \mathrm{~N}$ has its most intense rotational transitions in our $3 \mathrm{~K}$ molecular beam) a frequency search of $1 \mathrm{MHz}$ or less was required. The empirical predictions are at least an order of magnitude better than the best theoretical

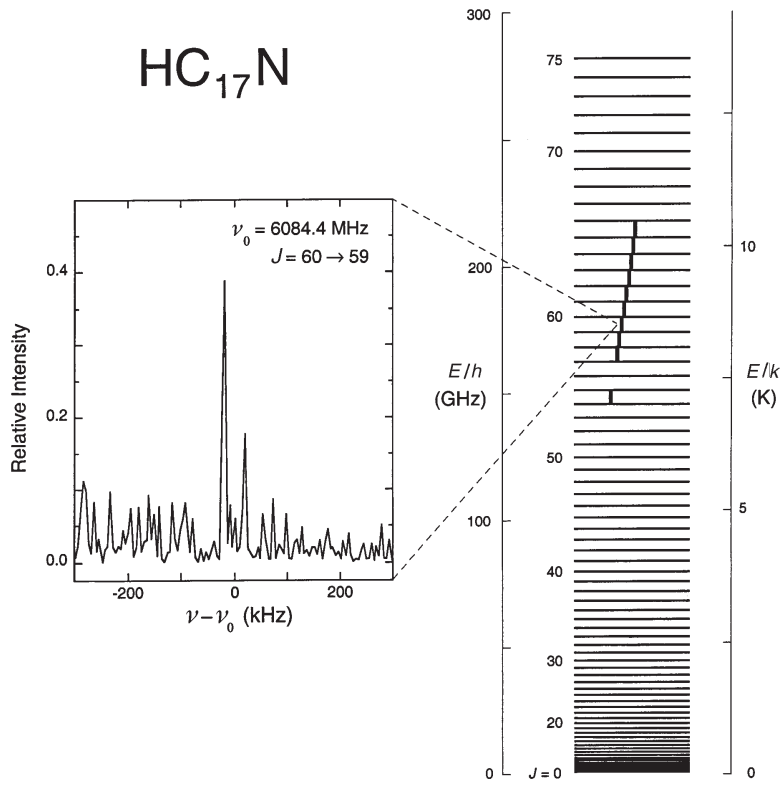

Fig. 4 Lower rotational levels of $\mathrm{HC}_{17} \mathrm{~N}$ with observed transitions indicated (vertical bars). Insert-Sample line of $\mathrm{HC}_{17} \mathrm{~N}$, obtained with an integration time of $60 \mathrm{~min}$. The double peaked line shape is instrumental in origin: it results from the Doppler shift of the supersonic molecular beam relative to the two traveling waves that compose the confocal mode of the Fabry-Perot cavity.

ab initio predictions, which are no better than $0.1 \%$ in accuracy. The close agreement of extrapolation with experiment suggests that the moment of inertia of these long chains is quite insensitive to structural details such as the rotation-vibration interaction which are important for small molecules. Little frequency search should therefore be required to detect $\mathrm{HC}_{19} \mathrm{~N}$ and $\mathrm{HC}_{21} \mathrm{~N}$, whose rotational constants are predicted to be only 37.069(2) $\mathrm{MHz}$ and 29.919(3) MHz, respectively.

The detection of $\mathrm{HC}_{17} \mathrm{~N}$ illustrates why it is worth refining the sensitivity of FTM spectroscopy. The lines of $\mathrm{HC}_{17} \mathrm{~N}$ are nearly an order of magnitude stronger than predicted by the nearly constant decrement in intensity observed for $\mathrm{HC}_{3} \mathrm{~N}$ to $\mathrm{HC}_{9} \mathrm{~N}$, and there is apparently almost no decrement in the actual number density of the longer chains in our beam-as Fig. 5 shows, $\mathrm{HC}_{17} \mathrm{~N}$ is about as abundant as $\mathrm{HC}_{15} \mathrm{~N}$. The longest cyanopolyynes are evidently more abundant and more easily detected than might have been expected, and still longer ones are probably within our grasp with slight improvement in sensitivity. In the interstellar gas, as in our molecular beam, one suspects that long chains because of their stability may be similarly more abundant than extrapolation from the shorter ones suggests.

An interesting and useful property of carbon chains is their extremely simple and predictable behavior under centrifugal distortion. Most polyatomic molecules deform on rotation in a fairly complicated way, characterized by a series of centrifugal distortion parameters which are not readily calculated from the structural parameters of the molecule, but must be empirically determined. We find in contrast that the cyanopolyynes and other carbon chains deform under centrifugal distortion like thin classical rods, with a Young's modulus that is independent of length. ${ }^{21}$ All carbon chains we have studied apparently distort under rotation in this simple way, regardless of either terminal groups or the low-lying vibrational structure of the individual molecules, and with a Young's modulus that is about the same for all. One might expect such classical behavior for long chains, but it is somewhat surprising that it holds for quite short ones, where quantum effects and the details of the vibrational structure of the molecule might be thought to be important.

Astronomical detection of molecules as large as $\mathrm{HC}_{17} \mathrm{~N}$ and perhaps even larger is now possible. Larger and better telescopes, such as the Green Bank 100 meter telescope and the 


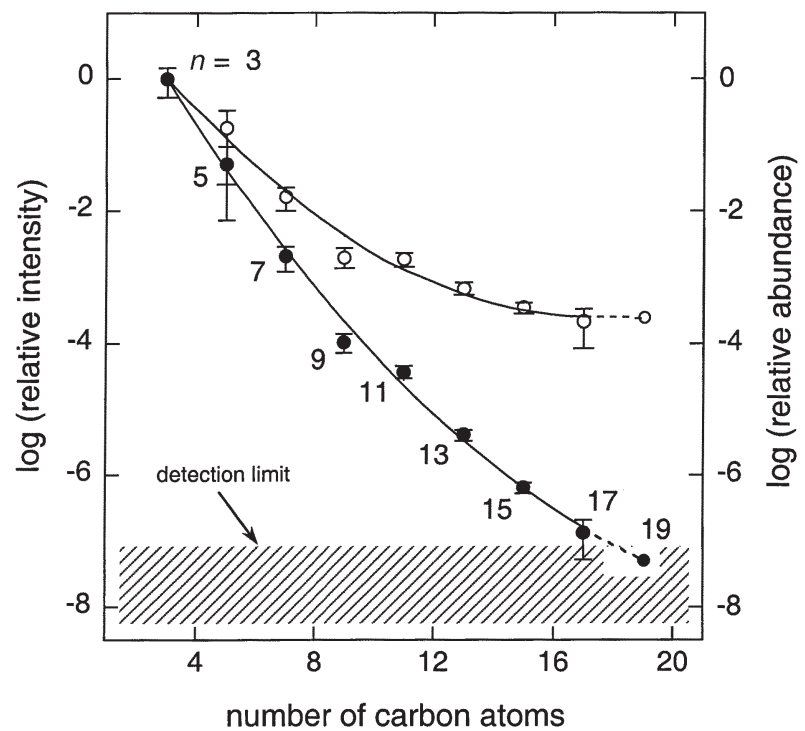

Fig. 5 Relative intensity of the strongest rotational lines of the cyanopolyynes (filled circles) and their relative abundance (open circles) in the FTM spectrometer, as a function of the number of carbon atoms in the chain. The error bars are estimated $2 \sigma$ uncertainties. Relative abundances are obtained from line intensities by taking into account the dependence on the chain length of the rotational partition functions and dipole moments. The limit of the detection sensitivity is approximately that achieved in a total observation time of $3 \mathrm{~h}$

resurfaced Arecibo 305 meter telescope, will soon be available in the $1-10 \mathrm{GHz}$ frequency range where the most intense astronomical lines of the longer chains lie. Although many hours of integration were required to detect lines of $\mathrm{HC}_{11} \mathrm{~N}$ in a particularly rich molecular source (the molecular cloud TMC-1 in the Taurus cloud complex), 22 considerably less time may be necessary to detect other long chains in this source with these next generation telescopes. Because small-scale unresolved structures may possess the highest column density of molecules in TMC-1,23 the new large telescopes with higher angular resolution may be considerably more efficient for astronomical detection.

Detection of the lowest frequency bending modes of long conjugated carbon chains may provide another way to observe molecules as large as $\mathrm{HC}_{17} \mathrm{~N}$ in astronomical sources. This approach has already been used with good success for short, non-polar chains such as $\mathrm{C}_{3}$ which have been detected in the laboratory 24 and in the circumstellar shell of the evolved carbon star IRC+10216 with the Infrared Space Observatory. ${ }^{25}$ The frequencies of the bending modes of a long chain such as $\mathrm{HC}_{17} \mathrm{~N}$ can be estimated to an uncertainty of about $15 \%$ from a semi-classical calculation in which bending modes scale in frequency as the inverse square of the chain. ${ }^{21}$ Applied to the two longest cyanopolyyne chains here, such scaling predicts 14 $\mathrm{cm}^{-1}(420 \mathrm{GHz})$ for the lowest bending mode of $\mathrm{HC}_{15} \mathrm{~N}$ and 11 $\mathrm{cm}^{-1}(330 \mathrm{GHz})$ for that of $\mathrm{HC}_{17} \mathrm{~N}$. Both frequencies are well within the range of existing high frequency radio telescopes, and the atmosphere is fairly transparent at these wavelengths. Precise laboratory measurements of the bending frequencies and accurate calculations of the transition moments are needed before undertaking such searches.

\subsection{Experimental structures of $\mathrm{HC}_{7} \mathrm{~N}, \mathrm{HC}_{9} \mathrm{~N}$, and $\mathrm{HC}_{11} \mathrm{~N}$}

The refinements in instrumental sensitivity and production that were required to detect $\mathrm{HC}_{17} \mathrm{~N}$ and other long polyyne chains now permit shorter ones to be observed with very high signalto-noise, such that all the isotopic species with a single carbon13 or nitrogen-15 are readily detected in natural abundance or with slight isotopic enrichment. For the cyanopolyyne series, these refinements have enabled us to determine precise effective $\left(r_{0}\right)$ structures for $\mathrm{HC}_{7} \mathrm{~N}, \mathrm{HC}_{9} \mathrm{~N}$, and $\mathrm{HC}_{11} \mathrm{~N}$-the largest reactive molecules to our knowledge for which accurate structures have been empirically derived. The structures of $\mathrm{HC}_{3} \mathrm{~N}$ (ref. 26) and $\mathrm{HC}_{5} \mathrm{~N}$ (ref. 27) were determined by isotopic substitution some time ago, so a detailed comparison of five successive cyanopolyynes can now be made to study how the chemical bonding and electronic structure vary as a function of chain length. For $\mathrm{HC}_{7} \mathrm{~N}$ and $\mathrm{HC}_{9} \mathrm{~N}$, ab initio values of the individual bond lengths have been calculated by Botschwina and co-workers ${ }^{28}$ allowing a direct comparison of experiment with theory.

For each cyanopolyyne, effective structures (Fig. 6) were determined from a least-squares fit of all the bonds in the
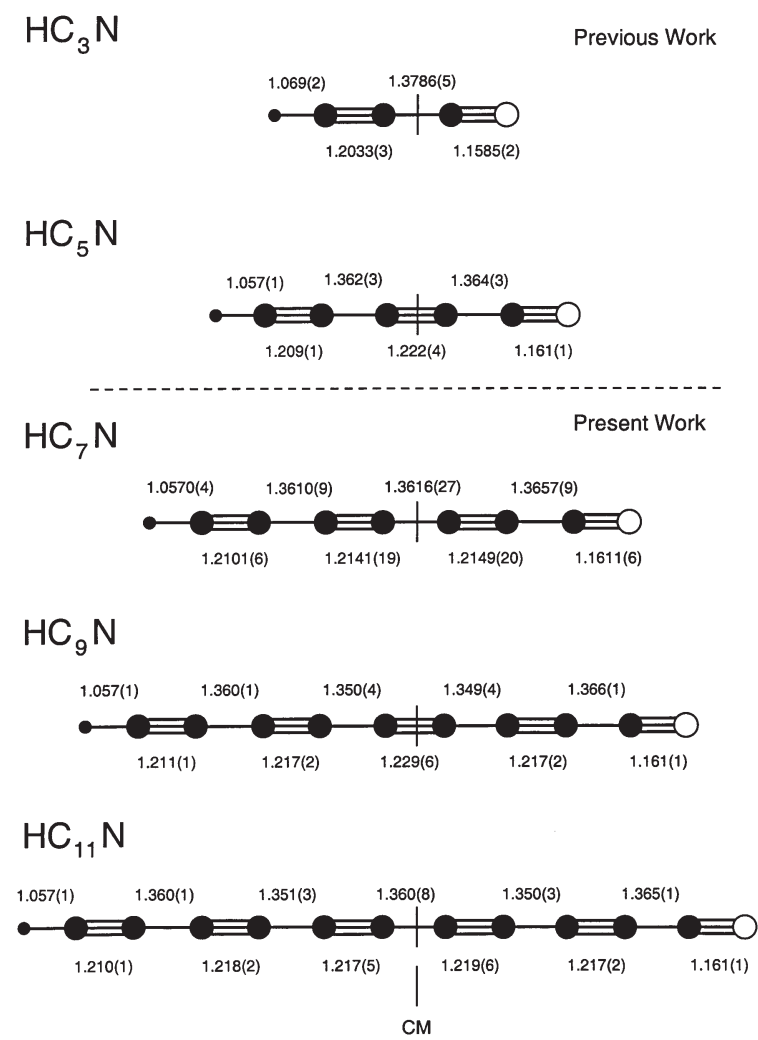

Fig. 6 Effective $\left(r_{0}\right)$ structures of $\mathrm{HC}_{3} \mathrm{~N}$ and cyanopolyynes up to $\mathrm{HC}_{11} \mathrm{~N}$ obtained by isotopic substitution. Uncertainties are in the units of the last significant digit.

molecule to the measured rotational constants (i.e., those of all the rare isotopic species plus the normal), on the assumption that each molecule is strictly linear. Toward the terminal atoms of each chain the bonds have been determined to remarkably high accuracy for molecules so large: of order $0.001 \AA$ or $0.1 \%$. Because the rotational constant of a linear chain is relatively insensitive to isotopic substitution near the center of mass, the central bonds are not so well determined, but are still good to about $0.01 \AA$ or $1.0 \%$. The experimental structures are in excellent agreement with recent high-level theoretical calculations, or, in the case of $\mathrm{HC}_{11} \mathrm{~N}$, with extrapolation from $\mathrm{HC}_{9} \mathrm{~N}$.

These structural determinations provide the first empirical evidence that well-defined bond alternation, as predicted by theory, exists throughout a long polyyne chain. There is no evidence in $\mathrm{HC}_{11} \mathrm{~N}$, for example, of a tendency of the central bonds to even out, approaching those of a cumulenic, double bonded structure, as observed for example in the shorter cumulenic chains such as $\mathrm{H}_{2} \mathrm{CCCC},{ }^{29}$ and predicted for longer members of that series. ${ }^{30}$ Presumably bond alternation persists in the limit of the infinite polyyne chain, as it does in the polyenes, the somewhat similar stable carbon chains with alternating single and double bonds, whose structures have been studied extensively by X-ray diffraction. ${ }^{31}$ With little or no 
additional effort, similar structural investigations to those described here could probably be undertaken for other polyyne chains such as methylpolyynes up to $\mathrm{H}_{3} \mathrm{C}_{11} \mathrm{H}$ and methylcyanopolyynes up to $\mathrm{H}_{3} \mathrm{C}_{9} \mathrm{CN}$. It is worth noting that although $\mathrm{HC}_{11} \mathrm{~N}$ was entirely unknown in the laboratory a short time ago, its ${ }^{13} \mathrm{C}$ isotopic species, less abundant relative to the normal species by a factor of 89 , can now be detected without isotopic enrichment.

\subsection{Reactive rings: rhomboidal $\mathrm{SiC}_{3}$}

A good example of the close interplay between laboratory astrophysics and astronomical observations is the recent discovery of rhomboidal $\mathrm{SiC}_{3}$, a highly polar, planar ring. Rhomboidal $\mathrm{SiC}_{3}$ is one of five known interstellar rings. Three of the four others $\left(\mathrm{C}_{3} \mathrm{H}_{2}, \mathrm{C}_{3} \mathrm{H}\right.$, and $\left.\mathrm{SiC}_{2}\right)$ are also highly reactive molecules generally unfamiliar to the laboratory chemist or physicist; the remaining ring, ethylene oxide $\left(\mathrm{C}_{2} \mathrm{H}_{4} \mathrm{O}\right)$, is a stable molecule. In the laboratory, as in the interstellar gas, the detection of rings has lagged behind detection of chains of similar size and composition in part because cyclic molecules lack simple harmonically-related rotational transitions that are readily spotted. The detection of new rings is worth pursuing because these molecules provide quite novel information on the rich and largely unexplored transition of carbon from chains to the two-dimensional graphitic lattice.

Rotational transitions of rhomboidal $\mathrm{SiC}_{3}$ and its rare isotopic species were first observed with our FTM spectrometer ${ }^{32}$ with a discharge through a dilute mixture of diacetylene and silanea very efficient method of production, it turns out, which yields high concentrations of $\mathrm{SiC}_{3}$ and longer linear silicon carbides up to $\mathrm{SiC}_{8}$. At most, four rotational transitions (Fig. 7) of each isotopic species lie within the frequency range of our spectrometer, but this number alone is sufficient to unambiguously establish the existence of this exotic ring, and to allow a precise determination of its geometry. If $\mathrm{SiC}_{3}$ were strictly planar and rigid, two parameters would suffice to determine the entire spectrum: three moments of inertia constrained by the Pythagorean theorem, but to describe the actual deformable molecule to the accuracy needed to conduct a radio astronomical search requires the inertial defect and several centrifugal distortion constants as well. Using the free-space millimeter-wave absorption spectrometer in this laboratory, a number of the higher rotational transitions of $\mathrm{SiC}_{3}$ were subsequently measured to within a few parts in $10^{7}$ up to $300 \mathrm{GHz}$, allowing the determination of the inertial defect and the six leading centrifugal distortion constants.

With the help of laboratory data, a number of millimeterwave rotational transitions of $\mathrm{SiC}_{3}$ have now been detected in IRC+10216 (Fig. 7) using the 12 meter NRAO telescope on Kitt Peak. ${ }^{33}$ The lines are fairly weak, but all have precisely the same radial velocity found for other molecules in this source, so the identification is beyond doubt. The rotational excitation of $\mathrm{SiC}_{3}$ is similar to that of $\mathrm{SiC}_{2}$, with a low rotational temperature $(10-20 \mathrm{~K})$ within the $K$-stacks, and a high rotational temperature (roughly $50 \mathrm{~K}$ ) across the $K$-stacks. The column density of $\mathrm{SiC}_{3}$ is perhaps $50 \%$ larger than that of $\mathrm{SiC}_{4}$.

Photochemical models of IRC $+10216^{34}$ predict that, as observed, $\mathrm{SiC}_{3}$ and $\mathrm{SiC}_{4}$ are more than two orders of magnitude less abundant than either $\mathrm{SiC}$ and $\mathrm{SiC}_{2}$. Some $\mathrm{SiC}_{3}$ is predicted from the photodissociation of $\mathrm{SiC}_{4}$. The implication is that the total amount of $\mathrm{SiC}_{3}$ cannot exceed that of $\mathrm{SiC}_{4}$. If $\mathrm{SiC}_{3}$ is found to be more abundant than $\mathrm{SiC}_{4}$, however, other reactions may be required to explain the observed abundance. To clarify the formation of $\mathrm{SiC}_{3}$ and to see if rhomboidal $\mathrm{SiC}_{3}$ is actually produced via photodissociation of linear $\mathrm{SiC}_{4}$, it would be desirable to detect additional $\mathrm{SiC}_{3}$ isomers in this astronomical source, and to compare their abundances. Two other low-lying, fairly polar $\mathrm{SiC}_{3}$ isomers, a triplet chain and second rhomboidal

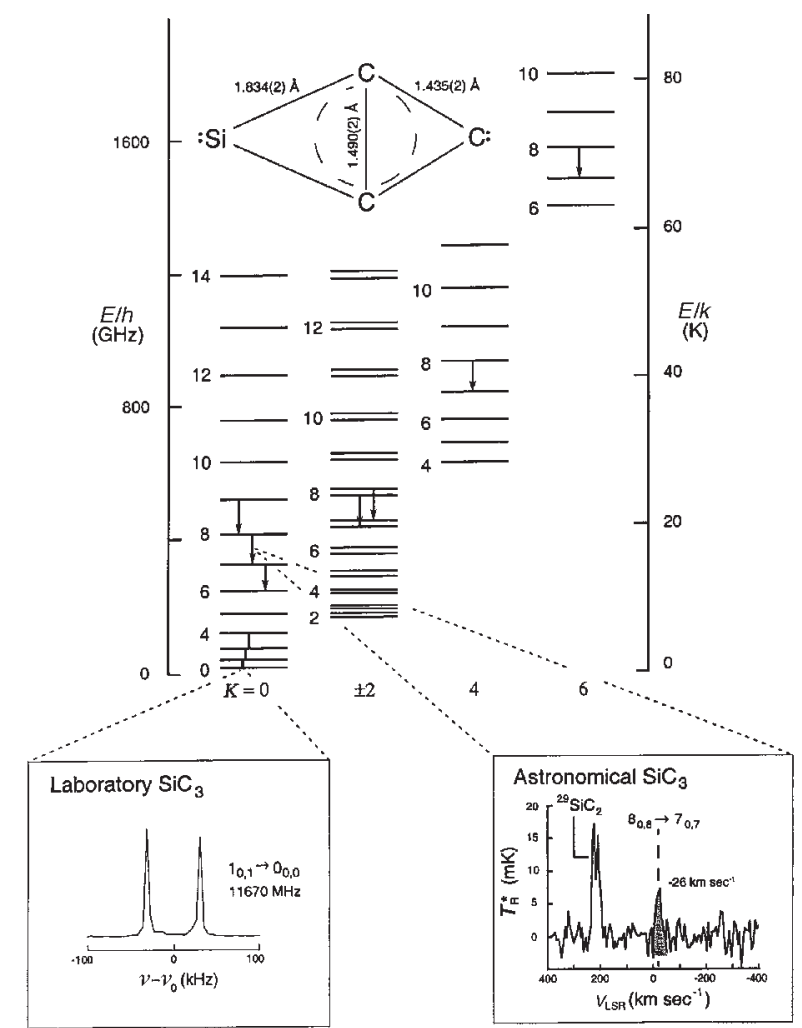

Fig. 7 Lower rotational levels of $\mathrm{SiC}_{3}$ showing the three transitions measured with the FTM spectrometer (vertical bars) and the seven transitions detected in IRC+10216 (lines with arrows). The effective $\left(r_{0}\right)$ structure obtained from isotopic substitution is shown above the energy level diagram. Inserts: (left) laboratory spectrum of the $1_{01} \rightarrow 0_{00}$ transition at $11,670 \mathrm{MHz}$, observed with a single gas pulse and an integration time of $0.2 \mathrm{~ms}$. The double peaked line shape as in Fig. 4 is instrumental in origin. (right) Radio spectrum of IRC+10216 near 91,845 MHz showing the $8_{08} \rightarrow$ $7_{07}$ transition. The spectral resolution is $2 \mathrm{MHz}$ and the integration time is $7 \mathrm{~h}$.

isomer, have recently been detected in this laboratory, so the data exists for a sensitive astronomical search.

\subsection{Nonpolar chains: linear triplet $\mathrm{HC}_{7} \mathrm{H}, \mathrm{HC}_{9} \mathrm{H}, \mathrm{HC}_{11} \mathrm{H}$, and $\mathrm{HC}_{13} \mathrm{H}$}

The first carbon chains detected with our CRD apparatus were the nonpolar radicals $\mathrm{HC}_{7} \mathrm{H}$ and $\mathrm{HC}_{9} \mathrm{H} .{ }^{35}$ For both the wavelengths of the strongest optical bands have been measured to an accuracy of about 1 part in $10^{6}$, and at least one vibronic band of each chain has also been measured to about the same level of accuracy. Because the origin bands of the two radicals have nearly the same intensity, and because the wavelengths of the strongest bands of each member in this homologous series are known fairly precisely from matrix spectra, ${ }^{36}$ it has now been possible to detect the gas-phase spectra of the next two odd-numbered chains $\mathrm{HC}_{11} \mathrm{H}$ and $\mathrm{HC}_{13} \mathrm{H} .{ }^{35}$ Sample spectra of each are shown in Fig. 8. For all four molecules, internal conversion seems to be much more rapid than radiative decay, and laser-induced fluorescence is not observed. For $\mathrm{HC}_{9} \mathrm{H}$, the origin band is close in wavelength to a weak diffuse band at $\lambda 5818$, but recent astronomical observations by McCall et al. ${ }^{37}$ indicate that the difference in wavelength between the two is too large to establish an identification.

Even though there is yet no evidence that these symmetric hydrocarbon radicals are carriers of the DIBs, they remain good candidates for astronomical detection in both the diffuse gas and in cool stellar atmospheres or circumstellar shells. The wavelength of the origin band of each chain can be determined very accurately from the laboratory data. High accuracy is possible because $\Sigma-\Sigma$ transitions lack both spin-orbit splitting 

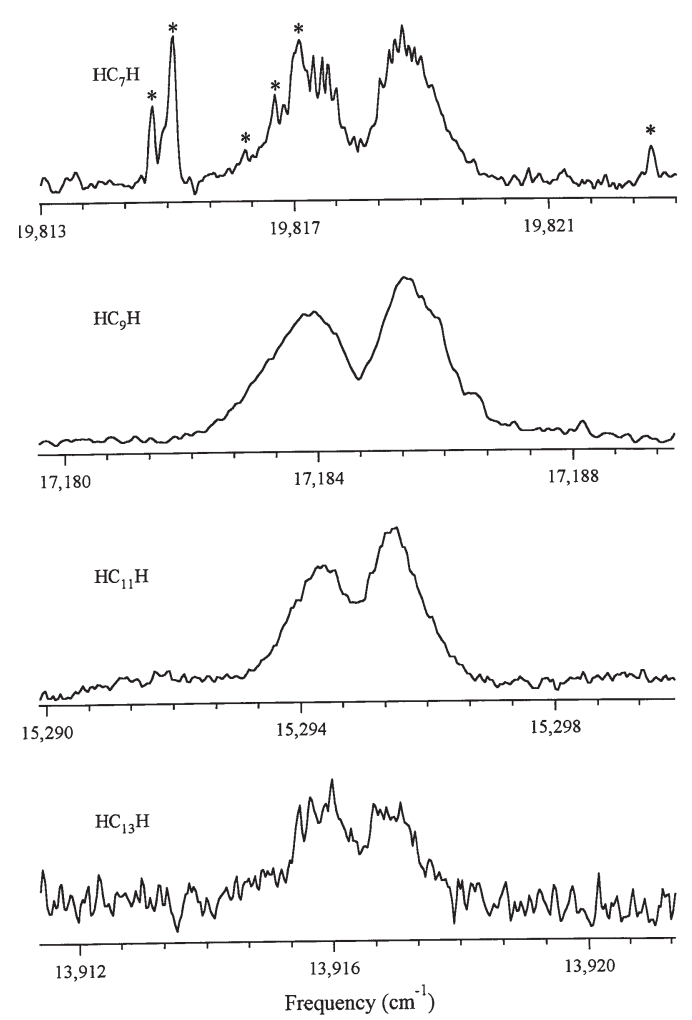

Fig. 8 Origin bands of $\mathrm{HC}_{7} \mathrm{H}, \mathrm{HC}_{9} \mathrm{H}, \mathrm{HC}_{11} \mathrm{H}$ and $\mathrm{HC}_{13} \mathrm{H}$. Features marked with an asterisk in the $\mathrm{HC}_{7} \mathrm{H}$ data indicate unrelated molecular lines, probably of $\mathrm{C}_{2}$, which appear in both normal and deuterated samples.

and red-shaded $R$-heads which tend to shift the wavelengths of electronic transitions by $0.05 \mathrm{~nm}$ or more depending on the rotational temperature either in the molecular beam or along different lines of sight in space. Since neither complication exists here, the band contours of the $\Sigma-\Sigma$ transitions are essentially insensitive to rotational temperature, and, as a result, the wavelengths of the origin bands can be determined about an order of magnitude more precisely than most $\Pi-\Pi$ transitions. ${ }^{38}$ If transitions of the symmetric chains can eventually be found in space, confirmation of the assignment might then come from detection of the characteristic $P$ - and $R$-branches in Fig. 8, or by detection of the somewhat weaker vibronic bands.

\subsection{A laboratory band at $\lambda 4429 \AA$}

Very recently a fairly strong molecular absorption band at 4429 $\pm 0.04 \AA$, closely centered on the strongest diffuse interstellar band at $4428.9 \pm 1.4 \AA$ has been detected. The laboratory band was found first in our supersonic molecular beam among the products of a discharge through benzene and toluene, then somewhat less intensely through diacetylene and other carbon chain sources. ${ }^{39}$ We have not yet been able to determine the exact elemental formula or structure of the carrier of the laboratory band, nor have we been able to demonstrate with certainty that the laboratory band is the same as that observed in space, but the agreement in wavelength between the two is so good (to a few parts in $10^{4}$ ) that a chance coincidence would seem unlikely. As Fig. 9 shows, the width of the laboratory band is significantly less than that of the interstellar diffuse band, but this difference is plausibly the result of the very low rotational temperature in the supersonic beam, possibly as low as $2 \mathrm{~K}$, relative to that of a nonpolar or weakly polar molecule in the diffuse interstellar gas (100-200 K).

The carrier of the laboratory band is almost certainly a hydrocarbon because the band is produced with different inert carrier gases, and is absent in all discharges studied so far through molecules containing oxygen, nitrogen, silicon, and sulfur. From the observed deuterium isotopic shifts we have
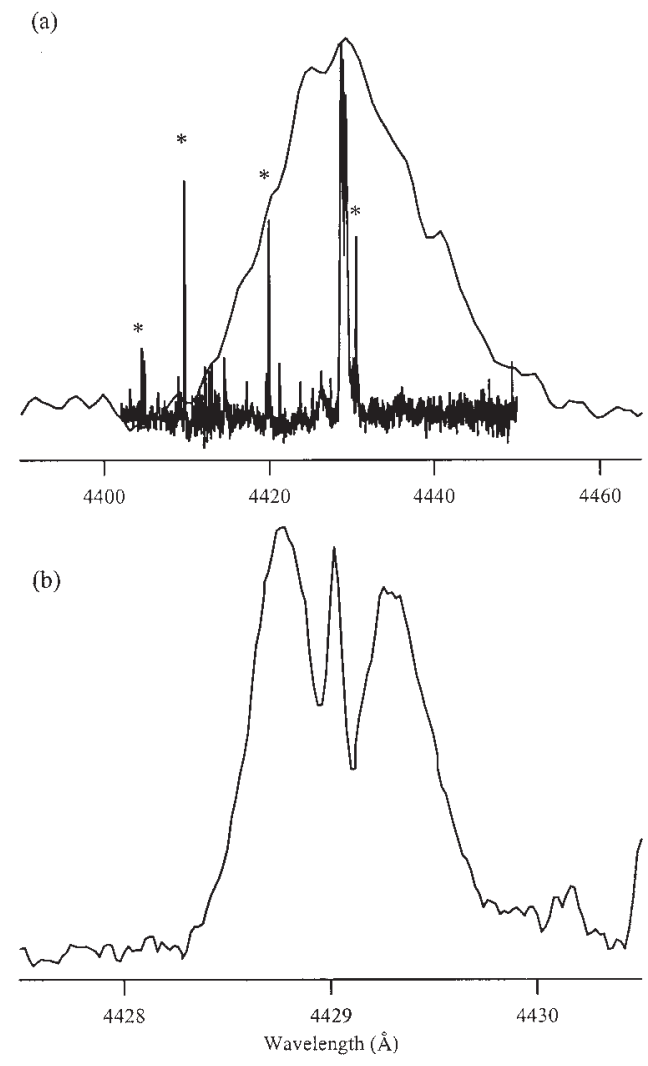

Fig. 9 (a) New laboratory molecular band, superposed on the strongest and best known interstellar band $\lambda 4429$, as observed towards a distant hot star. (b) The same laboratory band plotted on an expanded wavelength scale, displaying (from left to right) apparent $P$-, $Q$-, and $R$ - branches. Features marked with an asterisk denote unassigned lines of light hydrides.

established that the carrier probably has the elemental formula $\mathrm{C}_{n} \mathrm{H}_{5}$, and that the hydrogen atoms consist of two pairs of symmetrically-placed equivalent atoms plus a lone atom. The number of carbon atoms is likely to be in the 3-6 atom range because no plausible candidate carrier exists with two carbon atoms or less, and because we have found from our FTM studies that benzene discharges do not readily produce molecules with more than six carbon atoms. The number of plausible molecules (Fig. 9) in that mass range with the elemental formula of $\mathrm{C}_{n} \mathrm{H}_{5}$ is fairly small (Fig. 10), but includes at least two carbon chains,

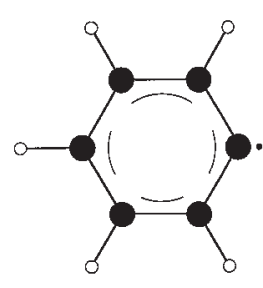

phenyl

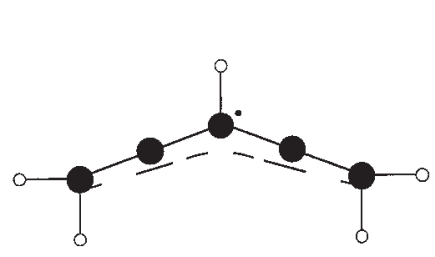

dimethylene allenyl

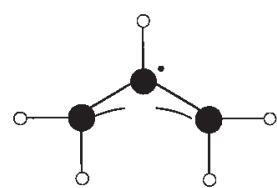

allyl

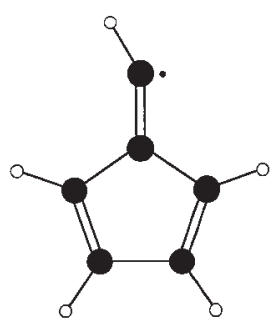

fulvenyl
Fig. 10 Selection of hydrocarbon molecules with the elemental formula $\mathrm{C}_{n} \mathrm{H}_{5}$ and the right symmetry to explain laboratory $\lambda 4429$.

allyl and dimethyleneallenyl radicals, and two cyclic aromatic hydrocarbons, the phenyl and fulvenyl radicals. Neutral allyl 
and phenyl would seem to be ruled out because their optical spectra are known, ${ }^{40,41}$ but the remaining two neutral radicals or positive or negative ions of all four can not be excluded. One of the more promising candidates is the dimethylene allenyl radical, $\mathrm{C}_{5} \mathrm{H}_{5}$, a slightly bent carbon chain which may be close enough to linear to reconcile the puzzling appearance of $P-, Q-$, and $R$-branches in the laboratory band with the five hydrogen atoms suggested from the isotopic shifts.

If the astronomical and laboratory bands are indeed the same, the present detection should constitute a substantial step towards the solution of the long standing problem of $\lambda 4428$. The array of laser, mass spectrometric, microwave, and theoretical techniques which can now be brought to bear on a problem of this kind is formidable, so an unambiguous assignment of the carrier of $\lambda 4428$ may now be possible on a fairly short time scale.

\section{Outlook}

In the radio band the laboratory astrophysics of molecular spectra is at last ahead of the radio astronomy, allowing new astronomical molecules to be found without searches in frequency that with large telescopes can be prohibitive in time and cost. Although many new chains and rings have been found with our present instrumentation, we are probably still very far from the optimal production efficiency for many of them and from the fundamental limits on the instrumental sensitivity. Continued experimentation with new precursor gases and different production methods such as laser ablation might yield significantly higher concentrations of the molecules of interest. Liquid helium cooling of the optics and first-stage of receiver amplification promises to improve the sensitivity by at least an order of magnitude, and a further improvement by a factor of perhaps five might be gained for molecules with ten or more heavy atoms by lowering the frequency of operation to the low$\mathrm{GHz}$ range, where lines are strongest at a rotational temperature of a few Kelvin. With modest and systematic improvements in both production and instrumental sensitivity, appreciably larger chains, rings, and molecular ions than those here may be within reach. The recent laser experiments here suggest that detection of the optical spectra of many of the radio molecules shown in Fig. 2 may now be possible; by extension, the same may be true for other known and postulated astronomical species. Such studies should provide a critical test of the hypothesis that carbon chains and other large organic molecules are the carriers of the optical diffuse interstellar bands, a problem in astronomical spectroscopy that has defied solution for over 60 years.

\section{Acknowledgements}

We thank our collaborators on the work under review here for many fruitful discussions, especially C. A. Gottlieb, A. J. Apponi, and C. D. Ball.

\section{References}

1 W. Klemperer, Annu. Rev. Phys. Chem., 1995, 46, 1.

2 N. Haider and D. Husain, J. Photochem. Photobiol., 1993, 70, 119.

3 R. I. Kaiser, D. Stranges, Y. T. Lee and A. G. Suits, Astrophys. J., 1997, 477, 982.

4 G. H. Herbig, Annu. Rev. Astron. Astrophys., 1995, 33, 19.

5 P. Jenniskens and F.-X. Desert, Astron. Astrophys., 1994, 106, 39.

6 M. Tulej, D. A. Kirkwood, M. Pachkov and J. P. Maier, Astrophys. J. Lett., 1998, 506, L69.

7 B. J. McCall, D. G. York and T. Oka, Astrophys. J., 2000, 531, 329

8 P. J. Sarre, J. R. Miles and S. M. Scarrott, Science, 1995, 269, 674.

9 A. E. Douglas, Nature, 1977, 269, 130.

10 F. Salama, E. L. O. Bakes, L. J. Allamandola and A. G. G. M. Tielens, Astrophys. J., 1996, 458, 621.

11 H. W. Kroto and M. Jura, Astron. Astrophys., 1992, 263, 209.

12 T. J. Balle and W.H. Flygare, Rev. Sci. Instrum., 1981, 52, 33

13 M. C. McCarthy, M. J. Travers, A. Kovács, C. A. Gottlieb and P. Thaddeus, Astrophys. J., Suppl. Ser., 1997, 113, 105.

14 J.-U. Grabow and W. Stahl, Z. Naturforsch., 1990, 45a, 1043

15 F. A. Jenkins, Y. K. Roots and R. S. Mulliken, Phys. Rev., 1932, 39, 16.

16 T. A. Dixon and R. C. Woods, J. Chem. Phys., 1977, 67, 3956.

17 A. O'Keefe and D. A. G. Deacon, Rev. Sci. Instrum., 1988, 59, 2544.

18 J. B. Paul and R. W. Saykally, Anal. Chem., 1997, 69, 287A.

19 M. C. McCarthy, J. U. Grabow, M. J. Travers, W. Chen, C. A. Gottlieb and P. Thaddeus, Astrophys. J. Lett., 1997, 494, L231.

20 T. Oka, J. Mol. Spectrosc., 1978, 72, 172.

21 P. Thaddeus, M. C. McCarthy, M. J. Travers, C. A. Gottlieb and W. Chen, Faraday Discuss., 1998, 109, 121.

22 M. B. Bell, P. A. Feldman, M. J. Travers, M. C. McCarthy, C. A. Gottlieb and P. Thaddeus, Astrophys. J. Lett., 1997, 483, L61.

23 W. D. Langer, T. Velusamy, T. B. H. Kuiper, S. Levin, E. Olsen and V. Migenes, Astrophys. J. Lett., 1995, 453, 293.

24 A. Van Orden and R. W. Saykally, Chem. Rev., 1998, 98, 2313.

25 J. Cernicharo, J. R. Goicoechea and E. Caux, Astrophys. J. Lett., 2000 , 534, L199.

26 J. K. G. Watson, A. Roytburg and W. Ulrich, J. Mol. Spectrosc., 1999, 196, 102.

27 A. J. Alexander, H. W. Kroto and D. R. M. Walton, J. Mol. Spectrosc., 1976, 62, 175.

28 P. Botschwina and M. Horn, J. Mol. Spectrosc., 1997, 185, 191, and references therein.

29 M. J. Travers, W. Chen, S. E. Novick, J. M. Vrtilek, C. A. Gottlieb and P. Thaddeus, J. Mol. Spectrosc., 1996, 180, 75.

30 S. A. Maluendes and A. D. McLean, Chem. Phys. Lett., 1992, 200, 511 .

31 B. Hudson and B. Kohler, Annu. Rev. Phys. Chem., 1974, 25, 437.

32 A. J. Apponi, M. C. McCarthy, C. A. Gottlieb and P. Thaddeus, J. Chem. Phys., 1999, 111, 3911, and references therein.

33 A. J. Apponi, M. C. McCarthy, C. A. Gottlieb and P. Thaddeus, Astrophys. J. Lett., 1999, 516, L103.

34 A. E. Glassgold and G. A. Mamon, Chemistry and Spectroscopy of Interstellar Molecules, ed. D. K. Bohme, E. Herbst, N. Kaifu and S. Saito, Univ. Tokyo Press, Tokyo, 1992, p. 261.

35 C. D. Ball, M. C. McCarthy and P. Thaddeus, J. Chem. Phys., 2000, 112, 10149, and references therein.

36 J. Fulara, P. Freivogel, D. Forney and J. P. Maier, J. Chem. Phys., 1995, 103, 8805 .

37 B. J. McCall, D. G. York and T. Oka, private communication.

38 T. Motylewski, Astrophys. J., 2000, 531, 312.

39 C. D. Ball, M. C. McCarthy and P. Thaddeus, Astrophys. J. Lett., 2000, 528, L61.

40 C. L. Currey and D. A. Ramsey, J. Chem. Phys., 1966, 45, 488

41 G. Porter and B. Ward, Proc. R. Soc. London, 1965, 287, 457. 